\title{
Colorectal Cancer Screening with Computed Tomography Colonography: Single Region Experience in Kazakhstan
}

\author{
Jandos Amankulov ${ }^{1,2}$, Dilyara Kaidarova ${ }^{3}$, Zhamilya Zholdybay ${ }^{2}$, Marianna Zagurovskaya ${ }^{4}$, Nurlan Baltabekov ${ }^{3}$, Madina \\ Gabdullina ${ }^{1}$, Akmaral Ainakulova ${ }^{1,2}$, Dias Toleshbayev ${ }^{1,2}$, Alexandra Panina ${ }^{1,2}$, Elvira Satbayeva ${ }^{5}$, and Zhansaya Kalieva ${ }^{6}$ \\ ${ }^{1}$ Department of Radiology and Nuclear Medicine, Kazakh Institute of Oncology and Radiology, Almaty, ${ }^{2}$ Department of Visual \\ Diagnostics, Asfendiyarov Kazakh National Medical University, Almaty, ${ }^{3}$ Department of Medical Oncology, Kazakh Institute of Oncology \\ and Radiology, Almaty, Kazakhstan, ${ }^{4}$ Department of Radiology, Medical College at the University of Kentucky, Lexington, KY, USA, \\ ${ }^{5}$ Center of Morphological Diagnostics, Kazakh Institute of Oncology and Radiology, Almaty, ${ }^{6}$ Department of Endoscopy, Kazakh Institute \\ of Oncology and Radiology, Almaty, Kazakhstan
}

Background/Aims: The aim of our study was to determine the efficacy of computed tomography colonography (CTC) in screening for colorectal cancer (CRC).

Methods: A total of 612 females and 588 males aged 45 to 75 years were enrolled in CTC screening. CTC was performed following standard bowel preparation and colonic insufflation with carbon dioxide. The main outcomes were the detection rate of CRC and advanced adenoma (AA), prevalence of colorectal lesions in relation to socio-demographic and health factors, and overall diagnostic performance of CTC.

Results: Overall, $56.5 \%$ of the 1,200 invited subjects underwent CTC screening. The sensitivity for CRC and AA was 0.89 and 0.97 , respectively, while the specificity was 0.71 and 0.99 , respectively. The prevalence of CRC and AA was 3.0\% (18/593) and 7.1\% (42/593), respectively, with the highest CRC prevalence in the $66-75$ age group ( $\geq 12$ times; odds ratio [OR], 12.11; 95\% confidence interval [CI], 4.45-32.92). CRC and AA prevalence were inversely correlated with Asian descent, physical activity, and negative fecal immunochemical test results $(\mathrm{OR}=0.43 ; 95 \% \mathrm{CI}, 0.22-0.83$; OR=0.16; 95\% CI, 0.04-0.68; OR=0.5; 95\% CI, 0.07-3.85, respectively). Conclusions: Our study revealed high accuracy of CTC in diagnosing colonic neoplasms, good compliance with CTC screening, and high detection rate of CRC. Clin Endosc 2022;55:101-112

Key Words: Cancer screening; Colonoscopy; Colorectal neoplasms; Computed tomography colonography

\section{INTRODUCTION}

Colorectal cancer (CRC) is one of the most frequently diagnosed malignancies worldwide, in both men and women.

Received: February 2, 2021 Revised: May 14, 2021

Accepted: May 18, 2021

Correspondence: Jandos Amankulov and Dilyara Kaidarova

Jandos Amankulov

Kazakh Institute of Oncology and Radiology, Abay Avenue 91, Almaty 050000, Kazakhstan

Tel: +8-727-2920061 (ext.196), Fax: +8-727-2920881, E-mail: zhandos.amankulov@gmail.com, ORCID: https://orcid.org/0000-0001-7389-3119

Dilyara Kaidarova

Kazakh Institute of Oncology and Radiology, Abay Avenue 91, Almaty 050000, Kazakhstan

Tel: +8-727-2920061 (ext.121), Fax: +8-727-3496116, E-mail: dilyara.kaidarova@ gmail.com, ORCID: https://orcid.org/0000-0002-0969-5983

(cc) This is an Open Access article distributed under the terms of the Creative Commons Attribution Non-Commercial License (http://creativecommons.org/ licenses/by-nc/3.0) which permits unrestricted non-commercial use, distribution, and reproduction in any medium, provided the original work is properly cited.
The highest incidence rates were registered in West European countries (up to 51.2/100,000), Australia/New Zealand (36.9/100,000), and North America (31.5/100,000). ${ }^{1}$ However, according to the International Agency for Research on Cancer (IARC), in some Asian countries (e.g., Republic of Korea, Japan, Singapore), the incidence rate might be as high as that in Western Europe. ${ }^{2}$ In addition, a mortality rate of 17.3 cases per 100,000 people is reported in Asia, which is higher than those in North America $(10.1 / 100,000)$ and Australia (10.9/100,000). High mortality rates have been reported even in some Asian countries with relatively low incidences of CRC (Brunei, 13.9/100,000; Armenia, 12.0/100,000; Malaysia, 11.2/100,000). Moreover, in recent years, there has been a steady increase in the incidence of CRC in the Asia-Pacific region. ${ }^{2}$

According to the GLOBOCAN 2018 database, Kazakhstan has the highest incidence $(15.4 / 100,000)$ and mortality $(10.5 / 100,000)$ rates of CRC among the countries of South 
and Central Asia. ${ }^{1}$ In Kazakhstan, CRC ranks fifth among all malignancies after breast, lung, cervical, and stomach cancers with more than 3,000 new cases registered annually. CRC is also the third most frequent cause of cancer related deaths after lung, breast, and stomach cancers with more than 2,000 mortality cases in 2018. ${ }^{3}$ Over the past 10 years, the incidence of CRC in the country has increased from 21.9 to 25.0 cases per 100,000 people, and IARC expects further growth in incidences over the next 20 years. Interestingly, lethal outcomes from CRC in the country have stabilized over the past decade (approximately 1,500 deaths per year)., ${ }^{1,3}$

It is believed that slow-growing colorectal polyps precede the development of CRC, with the latter driven by multiple genetic and epigenetic alterations. ${ }^{4}$ Such a transformation usually takes about 10 years, thus emphasizing the pivotal role of early detection of pre-malignant polyps. ${ }^{5}$

Screening for CRC and colonic polyps can be achieved by a fecal immunochemical test (FIT), conventional colonoscopy (CC), or computed tomography colonography (CTC). Screening with CC reduces the CRC death rate by $68 \%$, with hemorrhage and perforation reported as the main complications in 0.3-3.2 and 0.1-2 cases per 1,000 studies, respectively. ${ }^{6,7}$ The Asia Pacific Working Group for CRC Screening recommends $\mathrm{CC}$ for screening only high-risk groups. ${ }^{8}$ The effectiveness of $\mathrm{CC}$ is reduced by contraindications to the test, its low availability, and suboptimal participation of the population. ${ }^{9,10}$

Analysis of the effectiveness of the CRC screening program in Kazakhstan for the first five years (from 2011 to 2015) showed a low adenoma detection rate (17\%) and moderate population coverage with CC in the FIT-positive population (72.5\%). ${ }^{11}$ Since 2011, the primary screening method for CRC in Kazakhstan is a FIT, followed by CC in positive cases.

Virtual colonoscopy or CTC has $88.8 \%$ sensitivity and 75.4\% specificity for the detection of CRC and adenomas larger than $6 \mathrm{~mm}$, with at least the same accuracy as CC. ${ }^{12}$ In addition, CTC has a lower radiation dose burden on the patient compared to barium enema $(2.17 \pm 0.12$ milliSievert [mSv] vs. $4.12 \pm 0.17 \mathrm{mSv}){ }^{13}$ Moreover, minimal invasiveness, safety, and shorter procedure time in comparison with $\mathrm{CC}$ are additional benefits of CTC..$^{14,15}$ The US Preventive Services Task Force have included CTC as one of the primary CRC screening options. ${ }^{16}$ Furthermore, the European Society of Gastrointestinal Endoscopy and the European Society of Gastro-Abdominal Radiology recommend the use of CTC where there is no organized FIT-based population CRC screening program. ${ }^{17}$ However, the Asia Pacific Working Group for CRC Screening does not recommend CTC as a primary screening tool, citing a weak evidence base in the region. ${ }^{8}$

The main goals of this study were (i) to determine the de- tection rate of CRC and colonic advanced adenoma (AA) by CTC in asymptomatic populations of a single geographic region in the country of Kazakhstan, (ii) to establish the value of $\mathrm{CTC}$ in the diagnosis of CRC and AA, and (iii) to investigate the association of colorectal adenoma and CRC with sociodemographic and health factors.

\section{MATERIALS AND METHODS}

\section{Study design and population}

This single-center prospective observational study was approved by the local ethics committee, and informed consent was obtained from all participants.

A minimum of 384 participants was required to achieve a study power greater than $95 \%$, according to our sample calculation, which was based on a $25 \%-34 \%$ participation rate for CRC screening with CTC reported in a previous study. ${ }^{18}$

General practitioners (GPs) engaged in our study selected 3,000 subjects who met the eligibility criteria by analyzing their medical records. The inclusion criteria were asymptomatic individuals aged 45-75 years with no personal history of CRC and/or colonic AA. The exclusion criteria included inflammatory bowel diseases (e.g., Crohn's disease or ulcerative colitis, diverticulitis requiring treatment), pregnancy, previous colonoscopy, or CTC within five years prior to the study. Among 3,000 eligible subjects, 1,200 residents of the Almaty region, including the city of Almaty (Kazakhstan), were selected using a computer-generated random allocation sequence. GPs sent invitation letters to all 1,200 selected individuals between June 2016 and July 2019, inviting them to undergo CTC screening at the Kazakh Institute of Oncology and Radiology (Almaty, Kazakhstan), the leading tertiary cancer center of the country. The invitation letters comprised detailed information on CTC (including benefits and potential complications), and institution-based study-specific contact phone numbers for any questions and appointment schedules.

\section{Patient preparation and CTC exam}

Colon cleansing was achieved by full cathartic preparation with polyethylene glycol (PEG)-based solution as described in a previous publication, ${ }^{19}$ but with no dietary restrictions. To tag residual fluid and stool, $20 \mathrm{~mL}$ of iodinated contrast agent was administered at least three hours prior to the examination, unless there was a medical contraindication.

CTC was performed at the Kazakh Institute of Oncology on 64-slice CT scanners (Somatom Definition AS; Siemens, München, Germany and LightSpeed VCT; General Electric, Boston, USA) using a low-dose protocol (collimation $32 \times 0.6$, 
pitch 1.4 , rotation time $0.5 \mathrm{~s}, 120 \mathrm{kVp}, 50 \mathrm{mAs}$ ) in the supine and right decubitus positions. Colonic distension was achieved using an automatic insufflator (CO 2flow; Ulrich Medical, Ulm, Germany), with the degree of bowel distension assessed similar to the colon cleansing preparation. The Syngo VIA workstation (Siemens, München, Germany) was used for image processing, analysis, and clinical reporting. All CTC examinations were evaluated by two radiologists with three years of general CT experience and prior training in CTC, and two radiologists with $>10$ years and $\geq 200$ exam reads in CTC.

Analysis of CTC exams included a quick preview of a three-dimensional (3D) model of the colon, followed by the endoluminal fly through mode for primary search of colonic lesions with two-dimensional (2D) problem solving on the images obtained in both supine and right decubitus positions.

CTCs were considered positive if CRC or polyps $>6 \mathrm{~mm}$ were present. Colorectal lesions were typically visualized as exophytic masses or focal colonic wall thickening. The size (average, based on 2D and 3D measurements), morphology (sessile, pedunculated, or flat), location of the lesion, and distance from the anorectal junction were recorded. All positive CTC cases were evaluated by CC and, if endoscopically confirmed, underwent surgery or endoscopic polypectomy/biopsy; in all other cases, only endoscopy was offered. Relevant incidental extracolonic findings (ECF), such as vascular aneurysms and extracolonic mass lesions, have also been reported.

\section{Conventional colonoscopy}

Two endoscopists with six and 11 years of CC experience performed all CC examinations after bowel preparation with or without a low-fiber diet and using 2 L of PEG solution. In cases of incomplete colonoscopy due to poor preparation or pain, a new appointment was scheduled. CC was considered complete if visualization of the surface of all colonic segments was achieved. For the best correlation, CC findings were described in similar details and locations as in CTC. All detected lesions were either completely removed or biopsied for histopathological analysis during the procedure.

\section{Pathology}

Based on the histopathology, all colonic lesions were adenomatous (with dysplasia grading and assessment of villous component), hyperplastic, hamartomatous, or carcinoma according to the World Health Organization classification. ${ }^{20}$ Two experienced oncologic pathologists (with $\geq 10$ years of experience) blinded to the CTC and CC results evaluated the specimens using standard methods of specimen sampling and handling, lesion characterization, and reporting. ${ }^{21}$ The size of the lesions was measured in millimeters in three dimensions using a ruler, with the largest measurement selected for the final report.

\section{Data analysis}

SPSS software (version 21.0; IBM Co., Armonk, NY, USA) was used for all statistical analyses. All calculations were two-sided with statistical significance set as a $p$-value of $<0.05$. Characteristics of participants and colonic lesions are described as mean, standard deviation, and median values. The reliability of the results was assessed using the Mann-Whitney test and Wilcoxon test. Student's t-test was used to compare variables between groups. Cohen's kappa statistic was used to investigate the inter-rater agreement of lesion detection and characterization between radiologists, endoscopists, and pathologists.

\section{RESULTS}

\section{Participants' characteristics}

A total of 1,200 individuals (612 females, 588 males), aged 45 to 75 years, were invited to participate in the study. Out of these individuals, $548 \mathrm{did}$ not respond to the initial screening invitation; however, 26 of them accepted the invitation once they had a telephone conversation with the GPs. Overall, $56.5 \%(n=678)$ of the invitees accepted the CRC screening invitation. Among 678 study participants, 22 (3.2\%) did not comply with the test preparation for CTC, five (0.7\%) participants declined to complete the study due to abdominal pain during CTC, and 58 (8.6\%) did not adhere to the CC follow-up. Thus, 85 individuals with incomplete data were excluded from the study. Ultimately, data from 593 enrolled participants were subjected to statistical analysis (Fig. 1).

The mean age of the participants was $58.0 \pm 8.4$ years ( $58.3 \pm 8.6$ years for men and $57.9 \pm 8.2$ years for women). The sociodemographic and general health status characteristics of the participants are presented in Table 1 . The screening acceptance rate was higher in women, $63.4 \%$ (388 out of 612) vs. $34.9 \%$ for men (205 out of 588). Participation rate decreased with increasing age $(r=0.6138, p<0.05)$, with the highest rate being $38 \%$ of the $50-60$ age group. No serious adverse effects, such as bleeding or colonic perforation, were observed during CTC and/or CC.

\section{CTC vs. CC performance}

The duration of the CT scan in both the supine and right decubitus positions was 19.2 \pm 1.9 minutes ( $\mathrm{min}$ ) and was performed by highly skilled technologists with six years of related experience. The mean time radiologists spent to interpret a 


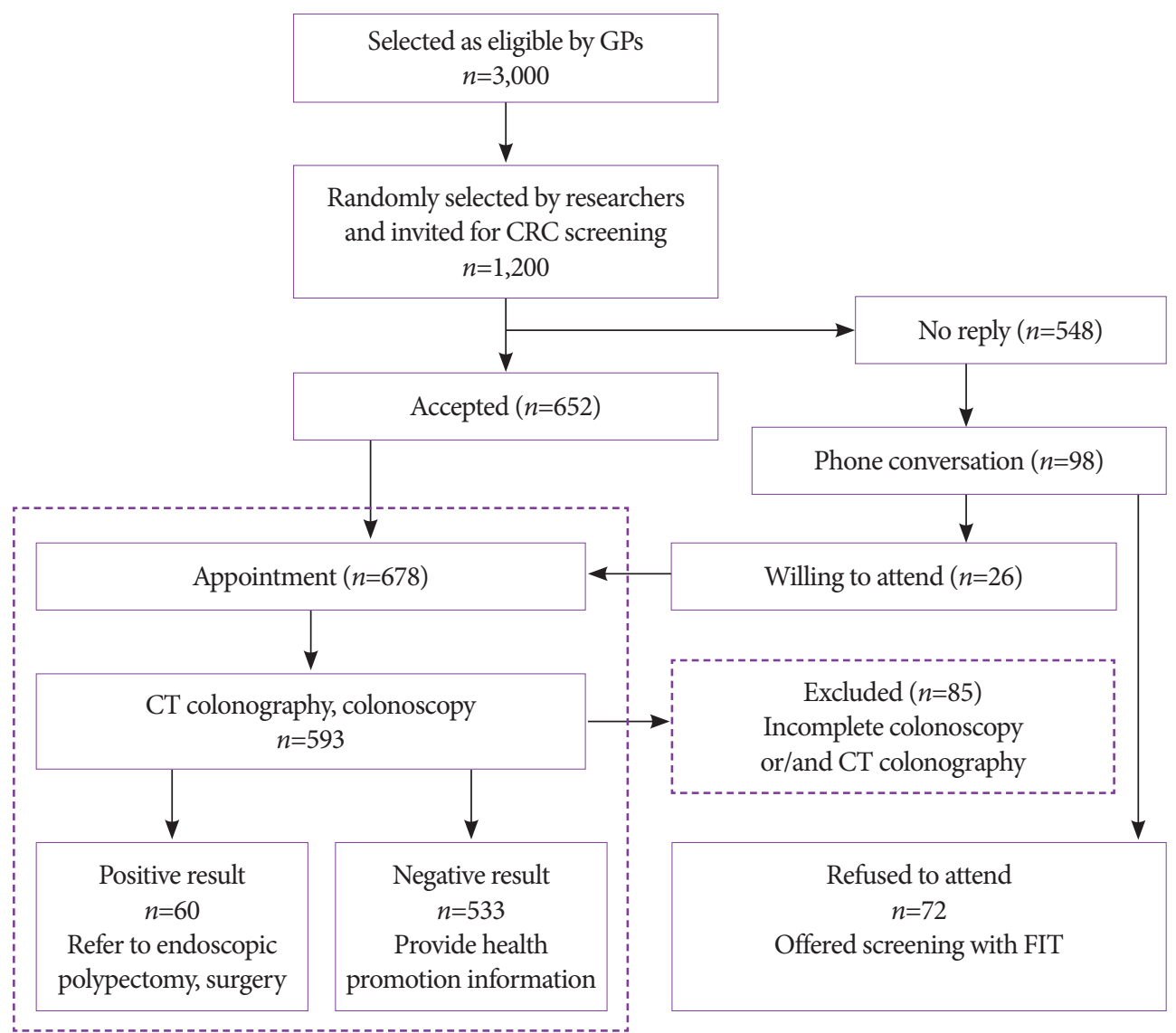

Fig. 1. Flowchart of participant recruitment algorithm. CRC, colorectal cancer; $\mathrm{CT}$, computed tomography; FIT, fecal immunochemical test; GP, general practitioner.

Table 1. Participants' Socio-Demographic and Health Status Characteristics

\begin{tabular}{lcc}
\hline & N & $(\%)$ \\
\hline Total & 593 & $(100)$ \\
Age (years) & & \\
$45-55$ & 264 & $(44.5)$ \\
$56-65$ & 200 & $(33.7)$ \\
$66-75$ & 129 & $(21.8)$ \\
Sex & & \\
Male & 205 & $(34.6)$ \\
Female & 388 & $(65.4)$ \\
Ethnicity & & \\
Native Kazakhs and non-Kazakh Asians & 453 & $(76.4)$ \\
Slavs & 140 & $(23.6)$ \\
Body mass index (BMI) $\left(\mathrm{kg} / \mathrm{m}^{2}\right)$ & & \\
Underweight and normal (BMI 18.4-24.9) & 259 & $(43.7)$ \\
Overweight (BMI 25.0-29.9) & 199 & $(33.6)$ \\
Obesity (BMI $\geq 30.0)$ & 135 & $(22.7)$ \\
\hline
\end{tabular}

CRC, colorectal cancer; FIT, fecal immunochemical test; N, number.

\begin{tabular}{|c|c|c|}
\hline & $\mathbf{N}$ & (\%) \\
\hline \multicolumn{3}{|l|}{ Physical activity (hours of dynamic activities per week) } \\
\hline $0-3$ & 390 & $(65.8)$ \\
\hline$\geq 3$ & 203 & $(34.2)$ \\
\hline \multicolumn{3}{|l|}{ Behavioral risk factors } \\
\hline None & 442 & $(74.5)$ \\
\hline Tobacco smoking & 96 & $(16.2)$ \\
\hline Alcohol consumption & 55 & $(9.3)$ \\
\hline \multicolumn{3}{|l|}{ Family history for cancer } \\
\hline First and second-degree relatives/CRC & 38 & $(6.4)$ \\
\hline First and second-degree relatives/non-CRC cancer & 195 & $(32.9)$ \\
\hline None & 360 & $(60.7)$ \\
\hline \multicolumn{3}{|l|}{ CRC screening with FIT } \\
\hline FIT-positive & 71 & $(12.0)$ \\
\hline FIT-negative & 61 & $(10.3)$ \\
\hline No previous participation in FIT-based CRC screening & 461 & $(77.7)$ \\
\hline
\end{tabular}


study was $13.9 \pm 5.9 \mathrm{~min}$, with longer interpretation for a less experienced ( $18 \pm 2.4 \mathrm{~min}$ ) vs. more experienced ( $\geq 10$ years of experience) radiologist ( $13 \pm 1.3 \mathrm{~min}, p=0.004)$. CC had a longer procedure time in our study than CTC $(45 \pm 4.1 \mathrm{~min})$, with a slightly shorter procedure time for an experienced $(35 \pm 3.8$ $\mathrm{min}$ ) vs. less experienced endoscopist ( $46 \pm 4.1 \mathrm{~min}, p=0.05$ ). Notably, the colonoscopies were performed without conscious sedation, which might have increased the total procedure time. The average waiting time for the $\mathrm{CC}$ procedure was 1.8 days (ranging from 0-5 days). The overall CC completion rate was $86 \%$.

The sensitivity of CTC for CRC vs. AA was 0.89 vs. 0.71, and the specificity was 0.97 vs. 0.99 , respectively (Table 2). CC demonstrated sensitivity and specificity for CRC comparable to CTC ( 0.88 and 0.98 , respectively) while having higher sensitivity $(0.84)$ and equivocal specificity $(0.99)$ for AA.

Kappa statistics indicated substantial agreement on CRC detection between three raters (radiologists, endoscopists, and pathologists) with a value of 0.61 . The agreement was most significant between radiologists and endoscopists (kappa value $0.68)$ and lower between radiologists and oncologic pathologists (0.43). The pooled agreement between all three groups of raters was weak for AA (0.39), with slightly better agreement between radiologists and endoscopists (0.47) than between radiologists and pathologists $(0.40)$. The degree of agreement stratified by experience was substantial among the radiologists and endoscopists ( 0.78 and 0.79 , respectively) and even higher for the pathologists $(0.88)$.

In addition, 22.3\% (132/593) of participants had attended the FIT-based National CRC screening program prior to selection for our study, with positive results in 71 (53.8\%) partic- ipants. The mean age for participants in the FIT-positive and negative groups was $59.1 \pm 7.0$ and $59.4 \pm 8.3$ years, respectively. There was a female predominance in both FIT groups $(0.73$ and 0.45 in FIT-positive and FIT-negative groups, respectively). CRC and AA were diagnosed in three (4.2\%) and in seven (9.9\%) participants, respectively, out of the 71 individuals with FIT-positive results. For the FIT-negative group $(n=61)$, CTC detected one case of CRC (1.6\%) and five $(8.2 \%)$ cases of AA. In the FIT-positive group, 24 out of 71 (33.8\%) participants declined endoscopy examination under the National CRC screening program, while simultaneously accepting CTC as a follow-up diagnostic tool in our study. In addition, all these individuals accepted CC when CRC or AA was diagnosed on CTC.

\section{Correlation of lesions by CTC Reporting and Data System (C-RADS) classification}

All CRC and colonic AA cases were histologically confirmed. The mean size of CRC was $6.1 \pm 2 \mathrm{~cm}$. Left colonic involvement was observed in $66.7 \%$ and $33.3 \%$ of the tumors in the proximal colon. The average size of the AA detected was $10 \pm 3 \mathrm{~mm}$. The most common AA type was sessile (76.2\%), followed by pedunculated and flat polyps ( $16.6 \%$ and $7.1 \%$, respectively). The left colonic distribution of AA was 2.5 times more frequent than right colon involvement $(71.2 \%$ vs. $28.6 \%$, respectively), with $33.3 \%$ of all AAs localized to the sigmoid colon.

Based on the C-RADS, ${ }^{22} \mathrm{C} 1$ category findings were present in 389 (65.6\%) out of 593 subjects (Figs. 2, 3), while C2 and C3 categories were present in 24/593 (4.0\%) and 39/593 (6.6\%) participants, respectively (Figs. 4, 5). Histopathological exam-

Table 2. Sensitivity, Specificity, Likelihood Ratios, and Predictive Values of Computed Tomography Colonography for Colorectal Carcinoma, Advanced Adenoma, and Both $(n=593)$

\begin{tabular}{|c|c|c|c|c|c|c|}
\hline & \multicolumn{2}{|c|}{ CRC } & \multicolumn{2}{|c|}{ AA } & \multicolumn{2}{|c|}{$\mathrm{CRC}$ and $\mathrm{AA}$} \\
\hline & Value & $95 \% \mathrm{CI}$ & Value & $95 \% \mathrm{CI}$ & Value & $95 \% \mathrm{CI}$ \\
\hline Sensitivity & $88.9 \%$ & $62.3-98.6 \%$ & $71.4 \%$ & $55.4-84.3 \%$ & $63.1 \%$ & $50.9-74.0 \%$ \\
\hline Specificity & $97.4 \%$ & $95.7-98.5 \%$ & $98.7 \%$ & $97.4-99.5 \%$ & $95.8 \%$ & $93.7-97.3 \%$ \\
\hline Positive LR & 34.1 & $20.2-57.6$ & 56.2 & $26.3-120.3$ & 14.9 & $9.5-23.2$ \\
\hline Negative LR & 0.1 & $0.03-0.4$ & 0.3 & $0.2-0.5$ & 0.4 & $0.3-0.5$ \\
\hline PPV & $51.3 \%$ & $38.4-64.1 \%$ & $81.6 \%$ & $67.4-90.5 \%$ & $62.6 \%$ & $51.7-72.3 \%$ \\
\hline NPV & $99.7 \%$ & $98.7-99.9 \%$ & $97.8 \%$ & $96.5-98.6 \%$ & $95.8 \%$ & $94.5-96.9 \%$ \\
\hline Accuracy & $97.1 \%$ & $95.5-98.3 \%$ & $96.7 \%$ & $95.0-98.0 \%$ & $92.5 \%$ & $90.0-94.5 \%$ \\
\hline Prevalence & \multicolumn{2}{|c|}{$3.0 \%$} & \multicolumn{2}{|c|}{$7.1 \%$} & \multicolumn{2}{|c|}{$10.1 \%$} \\
\hline
\end{tabular}

AA, advanced adenoma; CI, confidence interval; CRC, colorectal carcinoma; LR, likelihood ratio; NPV, negative predictive value; PPV, positive predictive value. 


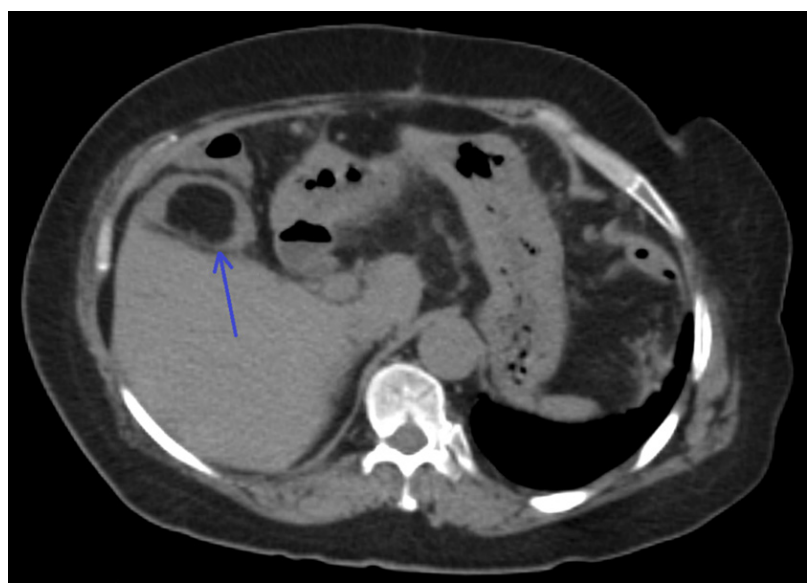

Fig. 2. Colonic lipoma, $\mathrm{C} 1$ category finding by computed tomography colonography reporting and data system. Axial computed tomography image showing a well-circumscribed fat density colonic lesion in the hepatic flexure (arrow). ination revealed that $7.1 \%(42 / 593)$ of all participants were diagnosed with AA. C4 category lesions (Fig. 6) were identified in 3.0\% (18/593); the mean age of the participants was $62.4 \pm 9.3$ years vs. $57.9 \pm 3.3$ years for $\mathrm{C} 1-\mathrm{C} 3$ category lesions. The age difference was less significant in cases of AA vs. participants without AA/CRC, $59.7 \pm 9.7$ years vs. $57.9 \pm 2.1$ years, respectively.

Linear association analysis between age and $\mathrm{C} 4$ category detection rate revealed a moderate positive relationship for both sexes $(\mathrm{r}=0.799 ; p \leq 0.05)$ with a stronger correlation for men vs. women ( $r=0.615$ vs. $r=0.012 ; p \leq 0.05)$. The correlation between age and AA detection rate demonstrated a positive relationship for females and for all participants $(r=0.521$ and $\mathrm{r}=0.395$, respectively) and a modest negative relationship for males $(r=-0.296)$. However, the analysis for AA did not reach statistical significance $(p>0.05)$.

The distribution of ECF was as follows: E0 in 0/593, E1 in
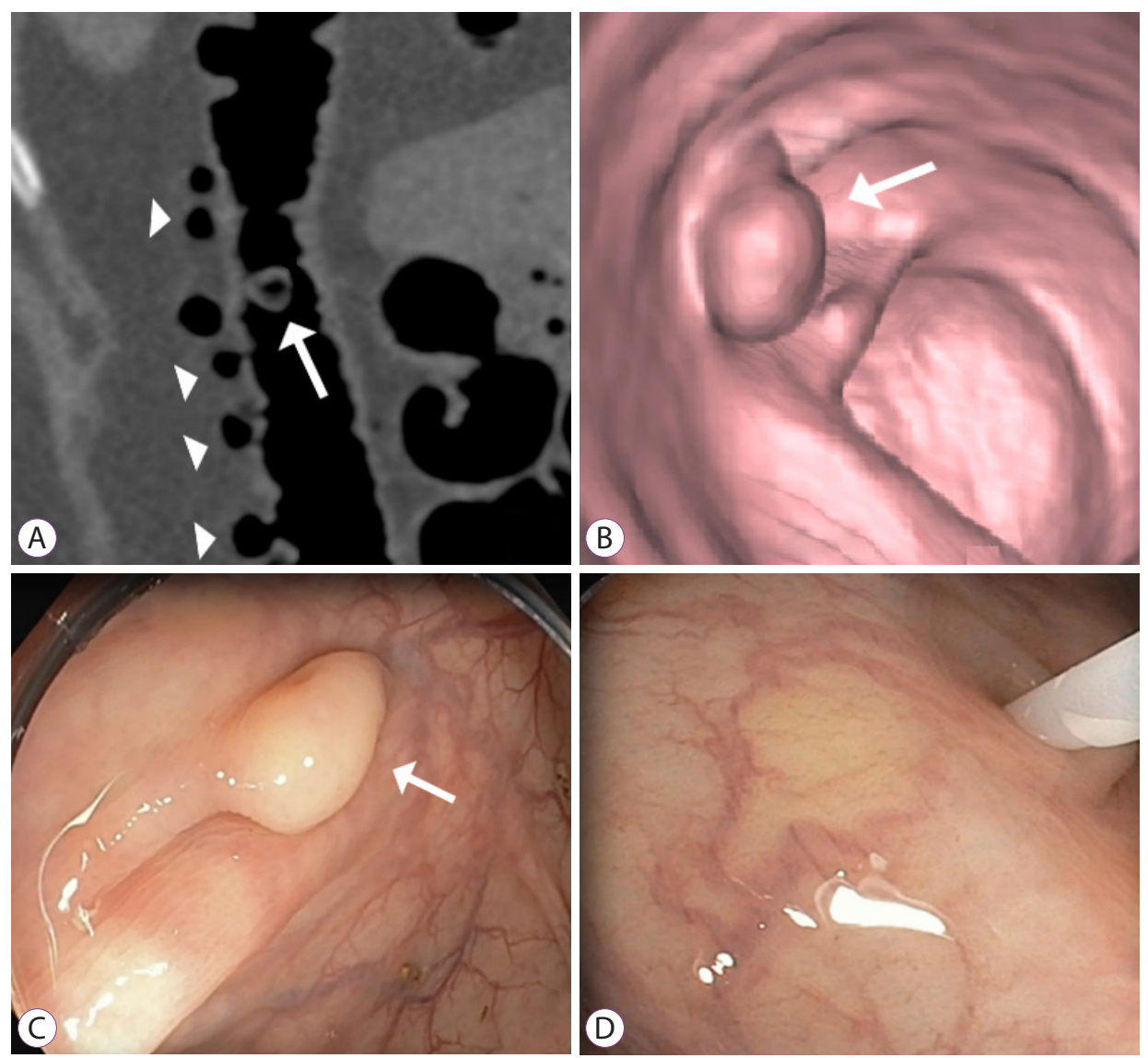

Fig. 3. Diverticulosis and inverted diverticulum, $\mathrm{C} 1$ category by computed tomography colonography reporting and data system. (A) Oblique reformatted computed tomography colonography (CTC) image demonstrates multiple bulging pouches in the colonic wall (arrowheads). One of the diverticula is inverted into the colonic lumen (arrow). (B) Endoluminal three-dimensional CTC image shows the inverted diverticulum as a polypoid lesion, without any mucosal changes in (C) endoscopic view (arrow). (D) Gentle pressure with closed biopsy forceps reverts the lesion to the typical diverticular appearance. 

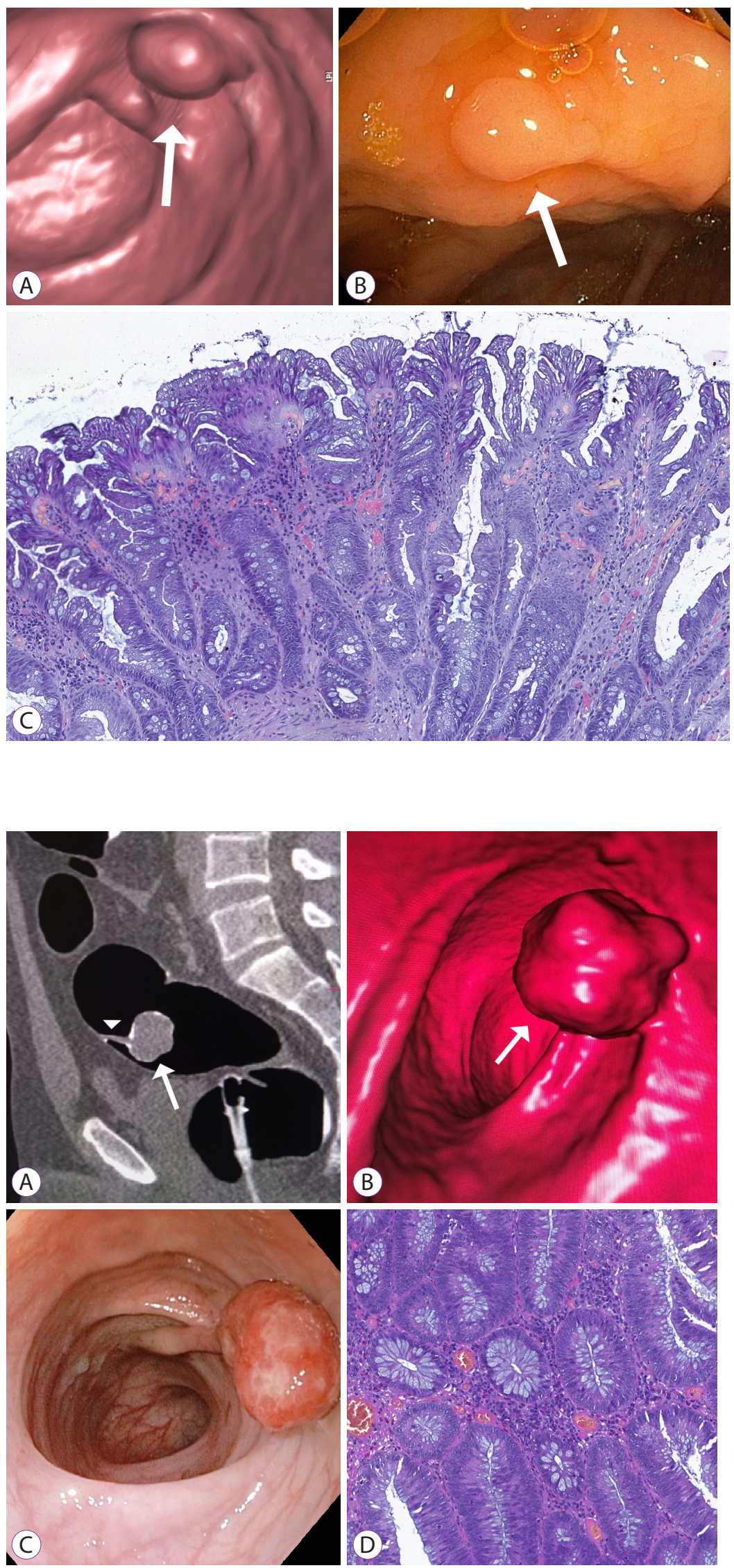

Fig. 4. Solitary colonic adenoma, C2 category by computed tomography colonography reporting and data system. (A) Endoluminal three-dimensional computed tomography colonography and (B) endoscopic images show $8 \mathrm{~mm}$ sessile colonic polyp (arrows). (C) Histologic evaluation represents a serrated polyp.
Fig. 5. Pedunculated adenoma of the upper rectum, C3 category by computed tomography colonography reporting and data system. (A) Sagittal two-dimensional and (B) endoluminal three-dimensional images of computed tomography colonography demonstrate $26 \mathrm{~mm}$ adenoma (arrows), connected to the mucosa by an elongated stalk (arrowhead). (C) Endoscopic image shows pedunculated adenoma with hyperemic and edematous mucosa. (D) Histologic evaluation reveals morphological changes consistent with severe dysplasia. 

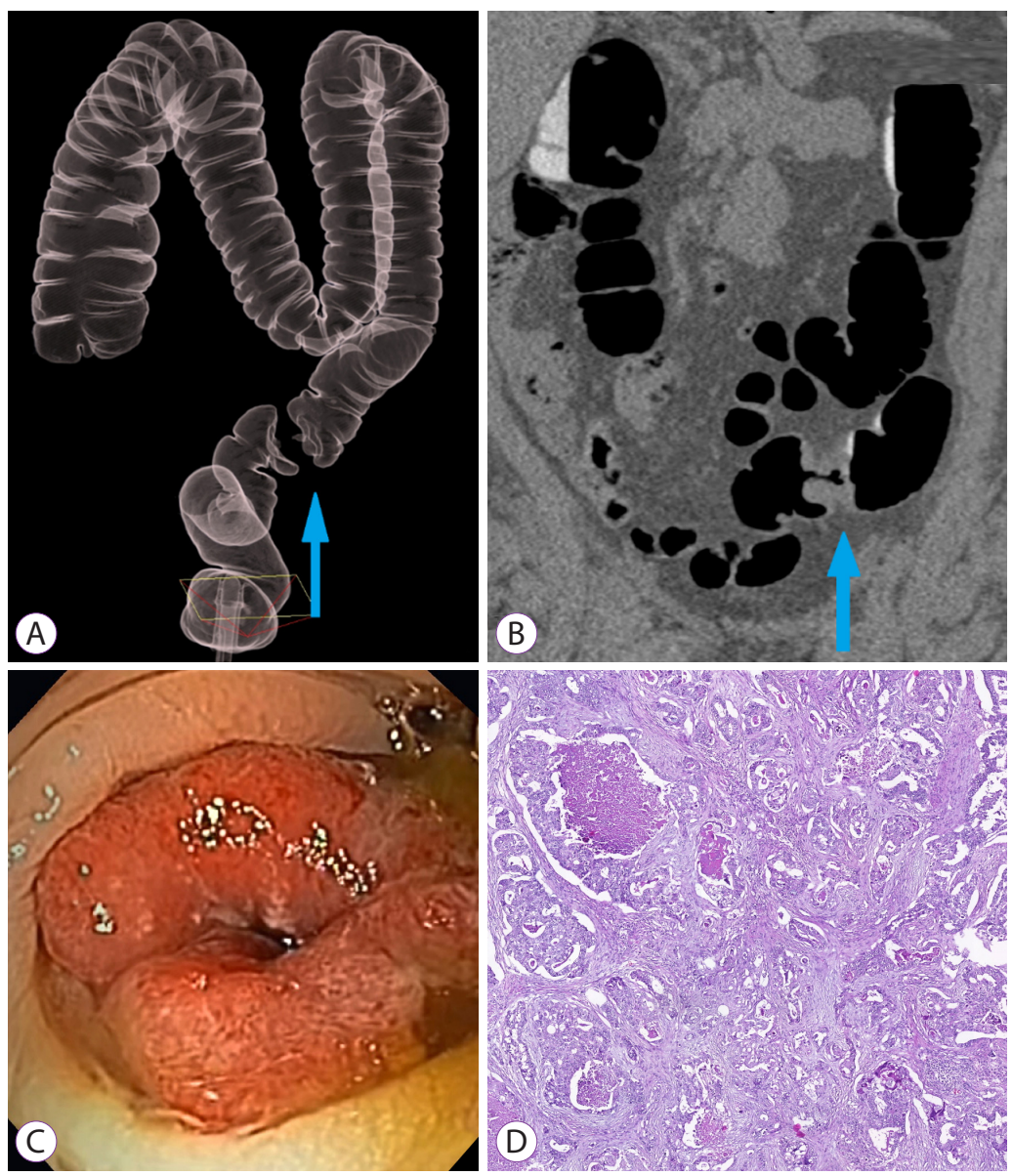

Fig. 6. Sigmoid colon carcinoma, $\mathrm{C} 4$ category by computed tomography colonography reporting and data system. Blue arrows on (A) three-dimensional and (B) coronal computed tomography colonography images indicate marked circumferential wall thickening of the sigmoid colon. (C) Endoscopic view reveals circumferential sigmoid tumor, with severe luminal compromise. (D) Histologic evaluation is positive for grade II adenocarcinoma.
$58.9 \%$ (349/593), E2 in 28.2\% (167/593), E3 in 11.5\% (68/593), and $\mathrm{E} 4$ in $1.5 \%$ (9/593) for all participants. The mean age of subjects with ECF was $59.4 \pm 8.8$ years. No linear correlation was observed between age and detection rates of the E2 and E3 categories. The age and rate of the E4 category were found to be moderately positively correlated $(r=0.461, p<0.01)$, with the highest rate of potentially important ECF in the 65-69 age group (37.5\% of all E4 cases). The rate of the E4 category was higher in men than in women by $82.4 \%$ ( $2.4 \%$ vs. $1.0 \%$, respectively), while the rate of $\mathrm{E} 3$ was $21.8 \%$ higher in men (13.2\% vs. $10.6 \%$, respectively). The most frequent reasons for inclusion of the E4 category included lesions suspicious for primary extracolonic malignancy (1.5\%) and aortic aneurysm $(0.2 \%)$.

\section{Odds ratio}

CRC was more than 12 times more prevalent in individuals older than 66 years (odds ratio [OR] $=12.11$; 95\% confidence interval [CI], 4.45-32.92, $p=0.01$ ), with less age-pronounced difference for AA compared to CRC (OR $=1.89$; 95\% CI, 0.67$3.71, p=0.05)$. The prevalence of CRC and AA in the native Kazakh and Asian populations was lower than that in the Slavic group $(\mathrm{OR}=0.43 ; 95 \% \mathrm{CI}, 0.22-0.83, p=0.01 ; \mathrm{OR}=0.61$; $95 \%$ CI, 0.22-1.65, $p=0.03$ ). Of note, the native Kazakh ethnicity comprised the vast majority of all Asian participants (92.0\%), while other Asian descents including Uygur, Korean, and Tatar nationalities comprised 3.7\%, 3.0\%, and 1.4\%, respectively.

Participants who were engaged in physical activity had at least four and two times lower risk of CRC and AA, respectively, than those who were physically inactive $(\mathrm{OR}=0.16 ; 95 \%$ CI, 0.04-0.68; $p \leq 0.05$ ). Participants were considered physically active if they engaged in weekly exercise of at least threehour dynamic activities such as fast walking, running, cycling, working out at the gym, aerobic dancing, playing football or tennis, etc. 
Table 3. Odds Ratio for CRC and Colonic AAAssociated with Participants' Socio-Demographic and Health Status

\begin{tabular}{|c|c|c|c|c|c|}
\hline Group categories & N (\%) & OR for CRC [95\% CI] & $p$ & OR for $\mathrm{AA}[95 \% \mathrm{CI}]$ & $p$ \\
\hline Total & $593(100)$ & & & & \\
\hline \multicolumn{6}{|l|}{ Age (years) } \\
\hline $45-55$ & $264(44.5)$ & $0.51[0.18-1.46]$ & & $0.84[0.44-1.59]$ & \\
\hline $56-65$ & $200(33.7)$ & $0.81[0.28-2.34]$ & & $0.68[0.33-1.38]$ & \\
\hline $66-75$ & $129(21.8)$ & $12.11[4.45-32.92]$ & 0.01 & $1.89[0.67-3.71]$ & 0.05 \\
\hline \multicolumn{6}{|l|}{ Sex } \\
\hline Male & $205(34.6)$ & $1.54[0.60-3.95]$ & & $0.74[0.37-1.18]$ & \\
\hline Female & $388(65.4)$ & $0.65[0.25-1.68]$ & 0.37 & $1.35[0.67-2.69]$ & 0.39 \\
\hline \multicolumn{6}{|l|}{ Ethnicity } \\
\hline Native Kazakhs and non-Kazakh Asians & $453(76.4)$ & $0.61[0.22-1.65]$ & & $0.43[0.22-0.83]$ & \\
\hline Slavs & $140(23.6)$ & $1.65[0.61-4.47]$ & 0.03 & $2.33[1.21-4.47]$ & 0.01 \\
\hline \multicolumn{6}{|l|}{ Body mass index (BMI) $\left(\mathrm{kg} / \mathrm{m}^{2}\right)$} \\
\hline Underweight and normal (BMI 18.4-24.9) & $259(43.7)$ & $1.64[0.64-4.21]$ & & $0.96[0.51-1.82]$ & \\
\hline Overweight (BMI 25.0-29.9) & $199(33.6)$ & $0.56[0.18-1.71]$ & & $0.99[0.51-1.92]$ & \\
\hline Obesity (BMI $\geq 30.0)$ & $135(22.7)$ & $0.97[0.31-2.99]$ & 0.31 & $1.07[0.51-2.23]$ & 0.86 \\
\hline \multicolumn{6}{|l|}{ Physical activity (hours of dynamic activities per week) } \\
\hline $0-3$ & $390(65.8)$ & $4.30[0.98-18.9]$ & & $2.33[1.06-5.13]$ & \\
\hline$\geq 3$ & $203(34.2)$ & $0.16[0.04-0.68]$ & 0.05 & $0.43[0.20-0.95]$ & 0.03 \\
\hline \multicolumn{6}{|l|}{ Behavioral risk factors } \\
\hline None & $442(74.5)$ & $0.88[0.31-2.53]$ & & $0.81[0.36-1.81]$ & \\
\hline Tobacco smoking & $96(16.2)$ & $1.50[0.48-4.66]$ & & $1.24[0.55-2.76]$ & \\
\hline Alcohol consumption & $55(9.3)$ & $0.57[0.07-4.34]$ & 0.58 & $0.11[0.01-1.88]$ & 0.13 \\
\hline \multicolumn{6}{|l|}{ Family history by cancer type } \\
\hline First and second-degree relatives / CRC & $38(6.4)$ & $0.86[0.11-6.61]$ & & $0.72[0.17-3.08]$ & \\
\hline First and second-degree relatives / non-CRC cancer & $195(32.9)$ & $1.02[0.38-2.76]$ & & $0.63[0.32-1.24]$ & \\
\hline None & $360(60.7)$ & $1.02[0.39-2.66]$ & 0.89 & $1.18[0.61-2.27]$ & 0.62 \\
\hline \multicolumn{6}{|l|}{ CRC screening results with FIT } \\
\hline FIT-positive & $71(12.0)$ & $1.49[0.42-5.27]$ & & $4.16[2.03-13.12]$ & \\
\hline FIT-negative & $61(10.3)$ & $0.50[0.07-3.85]$ & 0.04 & $0.21[0.03-1.61]$ & 0.01 \\
\hline No previous participation in FIT-based CRC screening & $461(77.7)$ & $1.01[0.33-3.10]$ & 0.99 & $1.01[0.33-3.10]$ & 0.99 \\
\hline
\end{tabular}

AA, advanced adenoma; CI, confidence interval; CRC, colorectal cancer; FIT, fecal immunochemical test; N, number; OR, odds ratio.

Statistically, a significant reduction in the probability of $\mathrm{CRC}$ and $\mathrm{AA}$ was observed in all participants with negative FIT results $(\mathrm{OR}=0.5 ; 95 \% \mathrm{CI}, 0.07-3.85, p=0.04)$. Sex-specific analysis of FIT positivity showed a two-fold lower probability of CRC (OR=0.65; 95\% CI, 0.25-1.68, $p=0.67)$ and an approximately 1.8 times higher detection rate of $\mathrm{AA}$ in women
$(\mathrm{OR}=1.35 ; 95 \% \mathrm{CI}, 0.67-2.69, p=0.39)$; however, the difference was not statistically significant. Despite variations (up to two times) in the likelihood of detection of CRC and AA between the other categories of participants, the differences were not statistically significant (Table 3 ). 


\section{DISCUSSION}

The success of a cancer screening program depends on the active enrollment of the population, sufficient acceptance rate of follow-up examinations, and timely health advice and/ or intervention. In our study, we achieved an overall good participation rate (56.5\%), with better engagement of women (63.4\%) and of the 50-60 age group for both sexes (38\% of all participants). These results indicate an improvement from the previously published data, where the overall participation rate of CTC screening was $25 \%{ }^{18,23}$ It should be noted, however, that participants' sociodemographic and health status characteristics, as well as bowel preparation techniques in these referenced trials, are different from ours.

A total of $12.5 \%$ ( 85 out of 678 ) enrolled participants were excluded from the study because of their inability to adhere to CC follow-up and/or bowel preparation for CTC. CC accounted for $86.2 \%$ of the included subjects. The reported acceptance rate of CC within the FIT-positive group under the National CRC screening program of Kazakhstan is between 59.3\%$76.2 \%{ }^{19}$ The highest reported colonoscopy follow-up rate of the eligible population was $97 \%$, reported by McNamara et al., ${ }^{24}$ with a pooled range of $52 \%-92.5 \%$ based on other studies results. ${ }^{25,26}$ In our study, among FIT-positive individuals, $33.8 \%$ (24 out of 71) of subjects were not compliant with CC follow-up under the National CRC screening program, while they were undergoing CTC. In addition, our study revealed another benefit of CTC, which was a shorter exam time (including interpretation), with an average of $33.1 \mathrm{~min}$ vs. 62.7 min for the total colonoscopy time.

Currently, adenoma detection rate is the main colonoscopy quality indicator. ${ }^{26}$ Among all endoscopically detected adenomas, the final histological diagnosis revealed AA in 2\%$18.3 \%$ of cases. ${ }^{27,28}$ Thus, the AA detection rate (7.1\%) in our study was similar to these published results. Interestingly, Karsenti et al. reported a strong positive relationship between AA detection rate and age among the participants of a similar age to our study. ${ }^{29}$ The AA detection rate was at least twice as high when the 40-44 age group was compared to the 45-49 age group, with no significant difference in the detection rate in comparison to the 50-54 age group. The AA detection rate was also quite similar in the 45-55 and 56-65 age groups but was more than 1.5 times lower in the $>66$ age group. The adenoma detection rate was not a quality indicator of the FIT-based National CRC screening program of Kazakhstan until 2018; consequently, there were no published data on adenoma and AA detection rates. The CRC detection rate was $3.0 \%$ in our study, with a lower detection rate in the $<50$ age group $(0.5 \%$ prevalence). The $2.5 \%$ detection rate for CRC in the $50-70$ age group was higher than that of the FIT-based CRC screening program in Kazakhstan (range of $0.02 \%-0.04 \%$ ).

CTC has achieved competitiveness in relation to endoscopy in terms of detection sensitivity for CRC and AA. Our data showed high sensitivity and specificity $(88.9 \%$ and $97.4 \%$, respectively) for CTC detection of CRC and moderate sensitivity and high specificity ( $71.4 \%$ and $98.7 \%$, respectively) for detection of advanced colonic adenoma. To date, CTC has passed through years of extensive trials and has been suggested as a safe and informative alternative to endoscopy. CTC in diagnosing CRC and colonic adenomas $>10 \mathrm{~mm}$ is considered as accurate as endoscopic evaluation, ${ }^{12}$ and the European Guidelines for Quality Assurance in CRC Screening has announced CTC as a new screening method. ${ }^{30}$ Similarly, CTC is a recommended screening option for CRC detection in the United States of America. ${ }^{16}$

Our demographic results revealed the prevalence of CRC and colonic AA in the Slavic population of Kazakhstan in comparison to the Asian descent. In previous studies, we have also noted higher CRC incidence in the ethnic Russian population of the country of Kazakhstan as well as in individual regions with a large proportion of Slavic inhabitants. ${ }^{31}$ This is concordant with previous data on low CRC prevalence in Asia when compared with that in Europe. ${ }^{32}$ However, among Asian countries, China, Japan, and Korea have exhibited the highest CRC detection rate, ${ }^{1}$ perhaps because the reasons for the above-mentioned disparities in CRC prevalence might not be attributed to only ethnic origin but rather reflect differences in socioeconomic status, behavioral, and individual factors.

We found that FIT-positive individuals have a 1.49- and 4.16-fold higher risk of being diagnosed with CRC and AA, respectively. Thus, a positive FIT test should prompt timely follow-up colonoscopy. Interestingly, the prevalence of CRC and AA may be high in FIT positive results even with previous negative colonoscopy results, according to the data from Kim et al. ${ }^{33}$ This study revealed a $5 \%$ and $20 \%$ prevalence of CRC and AA, respectively, with positive FIT results, suggesting the ability of FIT screening to potentially detect colonic lesions missed during colonoscopy. However, the corresponding rates among FIT-negative results were $1.9 \%$ and $10.3 \%$, respectively, indicating a considerably high rate of false-positive results with FIT screening. Kim et al. ${ }^{33}$ also reported a higher prevalence of AA than in our study ( $10 \%$ vs. $7.1 \%)$. These differences are likely due to the older age of participants in the referenced study (63.4 \pm 8.1 years) in comparison to ours $(58.0 \pm 8.4$ years).

Our results showed the protective effect of physical activity against $\mathrm{CRC}$ and colonic AA, similar to previous studies. In meta-analyses by Zhou et al. ${ }^{34}$ and Des Guetz et al., ${ }^{35}$ the 
physically active population had a lower risk of developing CRC. Moreover, physical activity was associated with a better prognosis for CRC and improved cancer-specific and overall survival.

The frequency of ECF is a great concern in CTC screening, which leads to increased costs and patient anxiety. A prior study highlighted up to $70 \%$ prevalence of ECF during CTC. ${ }^{36}$ However, Pooler et al. refuted this claim and stated that most of the ECFs are not relevant and require no further workup. ${ }^{37}$ Several other studies were congruent, with a wide range of E4 prevalence, from $2.1 \%-16 \% .{ }^{18,38}$ In comparison, the prevalence of the E4 category in our study was lower (1.5\%). We link such low indicators with the younger cut-off age of the participants in our study (45 years). The positive linear association between age and detection rate of clinically important ECF in our study was not unexpected. It is well known that age is one of the most studied risk factors not only for cancer but also for a wide range of infectious and non-infectious diseases. ${ }^{39}$

Our study had two limitations. The main limitation is the observational design, as randomized controlled trials are considered more preferable for evaluating the effectiveness of diagnostic methods. However, we compared the data from our study to the results from the FIT-based National CRC screening program when applicable. In addition, the overlapping of confidence intervals from observational and randomized studies is important, as it enhances the results.

Second, we mainly focused on the available acceptance rate for CTC and detection rates for CRC and AA when calculating the sample size. Consequently, the sample size was not large enough for statistical analysis of socio-demographic parameters.

CRC screening with CTC demonstrated a good participation rate in our single geographic region study. CTC has proven its accuracy in the diagnosis of both CRC and AA. CRC was diagnosed better with CTC than with the country's only FIT-based CRC screening program. Consistent with prior studies, older age and positive FIT-result were linked to higher $\mathrm{CRC}$ and $\mathrm{AA}$ incidences.

\section{Conflicts of Interest}

The authors have no potential conflicts of interest.

$$
\begin{aligned}
& \text { Funding } \\
& \text { None. }
\end{aligned}
$$

\section{Acknowledgments}

The views expressed in the submitted article are of the authors themselves and not an official position of their affiliated institutions and organizations.

\footnotetext{
Author Contributions

Conceptualization: Jandos Amankulov, Dilyara Kaidarova, Zhamilya
}

Zholdybay, Marianna Zagurovskaya, Nurlan Baltabekov, Madina Gabdullina, Akmaral Ainakulova, Dias Toleshbayev, Alexandra Panina, Elvira Satbayeva, Zhansaya Kalieva

Data collection: AP, MG

Formal analysis: NB

Funding acquisition: NB

Investigation: $\mathrm{ZK}, \mathrm{ES}$

Methodology: JA, MZ

Project administration: JA, NB

Resources: ES

Software: AA, DT

Supervision: DK, ZZ

Validation: DK

Visualization: ZZ

Writing-original draft: JA, MZ

Writing-review \& editing: JA, MZ

ORCID

Jandos Amankulov

Dilyara Kaidarova

Zhamilya Zholdybay

Nurlan Baltabekov

Marianna Zagurovskaya

Madina Gabdullina

Akmaral Ainakulova

Dias Toleshbayev

Alexandra Panina

Elvira Satbayeva

Zhansaya Kalieva

https:/orcid.org/0000-0001-7389-3119 https://orcid.org/0000-0002-0969-5983 https://orcid.org/0000-0003-0553-9016 https://orcid.org/0000-0002-7153-7409 https://orcid.org/0000-0002-3880-8051 https://orcid.org/0000-0003-4993-4747 https://orcid.org/0000-0003-1773-5145 https://orcid.org/0000-0002-7010-8776 https://orcid.org/0000-0002-3667-1872 https://orcid.org/0000-0002-1456-0047 https://orcid.org/0000-0003-0741-7354

\section{REFERENCES}

1. Bray F, Ferlay J, Soerjomataram I, Siegel RL, Torre LA, Jemal A. Global cancer statistics 2018: GLOBOCAN estimates of incidence and mortality worldwide for 36 cancers in 185 countries. CA Cancer J Clin 2018;68:394-424.

2. Pourhoseingholi MA. Increased burden of colorectal cancer in Asia. World J Gastrointest Oncol 2012;4:68-70.

3. Zhylkaidarova A, Kaidarova D, Batyrbekov K, Shatkovskaya O, Begimbetova $\mathrm{D}$. Trends of colorectal cancer prevalence in kazakhstan related to screening. Clin Endosc 2021;54:32-37.

4. Okugawa Y, Grady WM, Goel A. Epigenetic alterations in colorectal cancer: Emerging Biomarkers. Gastroenterology 2015;149:1204-1225. e12.

5. Rex DK, Ahnen DJ, Baron JA, et al. Serrated lesions of the colorectum: review and recommendations from an expert panel. Am J Gastroenterol 2012;107:1315-1329.

6. Kahi CJ. Reviewing the evidence that polypectomy prevents cancer. Gastrointest Endosc Clin N Am 2019;29:577-585.

7. Gupta S, Lieberman D, Anderson JC, et al. Recommendations for follow-up after colonoscopy and polypectomy: a consensus update by the us multi-society task force on colorectal cancer. Gastroenterology 2020;158:1131-1153.e5.

8. Sung JJY, Ng SC, Chan FKL, et al. An updated asia pacific consensus recommendations on colorectal cancer screening. Gut 2015;64:121-132.

9. Ojidu H, Palmer H, Lewandowski J, et al. Patient tolerance and acceptance of different colonic imaging modalities: an observational cohort study. Eur J Gastroenterol Hepatol 2018;30:520-525.

10. Abu-Freha N. [Should colonoscopy be the primary screening modality for colorectal cancer in israel?]. Harefuah 2019;158:523-528.

11. Kaidarova D, Zhylkaidarova A, Jumanov A, Shatkovskaya O, Mukhametbek B. Colorectal screening in kazakhstan: analysis of acces- 
sibility, problems, and prospects for further improvement. Oncol and Rad of Kazakhstan 2019;1:4-8.

12. Plumb AA, Halligan S, Pendsé DA, Taylor SA, Mallett S. Sensitivity and specificity of CT colonography for the detection of colonic neoplasia after positive faecal occult blood testing: systematic review and meta-analysis. Eur Radiol 2014;24:1049-1058.

13. Neri E, Faggioni L, Cerri F, et al. CT colonography versus double-contrast barium enema for screening of colorectal cancer: comparison of radiation burden. Abdom Imaging 2010;35:596-601.

14. Pickhardt PJ. Strong evidence in support of CT colonography screening. Lancet Oncol 2012;13:6-7.

15. Atkin W, Dadswell E, Wooldrage K, et al. Computed tomographic colonography versus colonoscopy for investigation of patients with symptoms suggestive of colorectal cancer (SIGGAR): a multicentre randomised trial. Lancet 2013;381:1194-1202.

16. US Preventive Services Task Force, Bibbins-Domingo K, Grossman DC, et al. Screening for colorectal cancer: us preventive services task force recommendation statement. JAMA 2016;315:2564-2575.

17. Spada C, Hassan C, Bellini D, et al. Imaging alternatives to colonoscopy: CT colonography and colon capsule. European Society of Gastrointestinal Endoscopy (ESGE) and European Society of Gastrointestinal and Abdominal Radiology (ESGAR) Guideline - Update 2020. Endoscopy 2020;52:1127-1141.

18. Sali L, Regge D. CT colonography for population screening of colorectal cancer: hints from European trials. Br J Radiol 2016;89:20160517.

19. Sali L, Grazzini G, Ventura L, et al. Computed tomographic colonography in subjects with positive faecal occult blood test refusing optical colonoscopy. Dig Liver Dis 2013;45:285-289.

20. Nagtegaal ID, Odze RD, Klimstra D, et al. The 2019 WHO classification of tumours of the digestive system. Histopathology 2020;76:182-188.

21. Salmo E, Haboubi N. Adenoma and malignant colorectal polyp: pathological considerations and clinical applications. EMJ Gastroenterol 2018;7:92-102.

22. Pooler BD, Kim DH, Lam VP, Burnside ES, Pickhardt PJ. CT colonography reporting and data system (C-RADS): benchmark values from a clinical screening program. AJR Am J Roentgenol 2014;202:1232-1237.

23. Zhu H, Li F, Tao K, et al. Comparison of the participation rate between CT colonography and colonoscopy in screening population: a systematic review and meta-analysis of randomized controlled trials. Br J Radiol 2020;93:20190240.

24. McNamara D, Qasim A, Lee N, Condon C, O'Morain C. Round one of the adelaide and meath hospital/trinity college colorectal cancer screening programme: programme report and analysis based on established international key performance indices. Ir J Med Sci 2011;180:549-552.

25. Poskus T, Strupas K, Mikalauskas S, et al. Initial results of the national colorectal cancer screening program in Lithuania. Eur J Cancer Prev
2015;24:76-80

26. Rex DK, Schoenfeld PS, Cohen J, et al. Quality indicators for colonoscopy. Gastrointest Endosc 2015;81:31-53.

27. Amano T, Nishida T, Shimakoshi H, et al. Number of polyps detected is a useful indicator of quality of clinical colonoscopy. Endosc Int Open 2018;6:E878-E884.

28. Klair JS, Ashat M, Johnson D, et al. Serrated polyp detection rate and advanced adenoma detection rate from a US multicenter cohort. Endoscopy 2020;52:61-67.

29. Karsenti D, Tharsis G, Burtin P, et al. Adenoma and advanced neoplasia detection rates increase from 45 years of age. World J Gastroenterol 2019;25:447-456.

30. Armaroli P, Villain P, Suonio E, et al. European code against cancer, 4th edition: cancer screening. Cancer Epidemiol 2015;39 (Suppl 1):S139S152.

31. Abdullayev MS, Nurgaziyev KS, Zhylkaydarova AZ, Mansurova AB. Epidemiological aspects of colorectal cancer in Kazakhstan. Nov Khirurgii 2017;25:394-403.

32. Siegel RL, Miller KD, Jemal A. Cancer statistics, 2019. CA Cancer J Clin 2019;69:7-34.

33. Kim NH, Jung YS, Lim JW, Park JH, Park DI, Sohn CI. Yield of repeat colonoscopy in asymptomatic individuals with a positive fecal immunochemical test and recent colonoscopy. Gastrointest Endosc 2019;89:1037-1043

34. Zhou XY, Yan L, Wang LL, Wang J. Association between physical activity and colorectal cancer risk and prognosis: A meta-analysis. Cancer Treatment and Research Communications 2016;9:62-69.

35. Des Guetz G, Uzzan B, Bouillet T, et al. Impact of physical activity on cancer-specific and overall survival of patients with colorectal cancer. Gastroenterol Res Pract 2013;2013:340851.

36. Veerappan GR, Ally MR, Choi J-HR, Pak JS, Maydonovitch C, Wong RKH. Extracolonic findings on CT colonography increases yield of colorectal cancer screening. AJR Am J Roentgenol 2010;195:677-686.

37. Pooler BD, Kim DH, Pickhardt PJ. Indeterminate but likely unimportant extracolonic findings at screening CT Colonography (C-RADS Category E3): incidence and outcomes data from a clinical screening program. AJR Am J Roentgenol 2016;207:996-1001.

38. Tutein Nolthenius CJ, Boellaard TN, de Haan MC, et al. Computer tomography colonography participation and yield in patients under surveillance for 6-9 $\mathrm{mm}$ polyps in a population-based screening trial. Eur Radiol 2016;26:2762-2770.

39. Johnston BC, Zeraatkar D, Han MA, et al. Unprocessed red meat and processed meat consumption: dietary guideline recommendations from the nutritional recommendations (NutriRECS) consortium. Ann Intern Med 2019;171:756-764. 